\title{
Spaces of Care and Graphic Medicine
}

\author{
Sathyaraj Venkatesan ${ }^{1} \&$ Livine Ancy $A^{2}$ \\ ${ }^{1}$ Associate Professor, Department of Humanities and Social Sciences, National Institute of \\ Technology, Trichy. Corresponding Author. Email: sathyaiitk@gmail.com \\ ${ }^{2}$ Research Scholar, Department of Humanities and Social Sciences, National Institute of \\ Technology, Trichy. Email: livine2212@gmail.com
}

\begin{abstract}
While there are several studies that focus on care settings in relation to verbal narratives, only a few studies have paid attention to how comics in general, and graphic medicine in particular, engage critical care environments and settings. Drawing strengths from the underground and alternative comics and capitalizing on health humanities, graphic medicine, a recent development in the comics genre, concentrates on the issues related to health, illness, and care. Coined by Ian Williams in 2007, graphic medicine refers to the intersection of comics and concerns of healthcare. Graphic medicine has always engaged informal, formal, and biomedical caregiving settings. Against this backdrop, the present article, drawing on relevant theoretical debates on spatial studies and care, examines Stan Mack's Janet\& Me (2004), Joyce Farmer's Special Exits (2014), and Sarah Leavitt's Tangles (2012). In so doing, the article seeks to delineate care facilities (family, hospitals, among others) and their impact on patients.
\end{abstract}

Keywords: graphic Medicine, informal care, hospital Care, institutional care, spaces of care.

\section{Philosophy of Care: An Introduction}

The term care has multiple shades of meaning. The proponents of care acknowledge that care is "the most deeply fundamental value" (Held, 2006, p. 17), characterising relational practices that aid in flourishing of human life. According to Benner et al., such relational practices "foster mutual recognition and realization, growth, development, protection, empowerment, and human community, culture, and possibility... relationships that are devoted... [to] assisting others to cope with their weaknesses, while affirming their strengths" (Benner et al., as cited in DeFalco, 2020, p. 34). Underlining care's ubiquity and its mercurial nature, Amelia DeFalco (2015), in her article Towards a Theory of Posthuman Care: Real Humans and Caring Robots, observes that: "Care is everyday and rarefied, professional and private, public and personal" (p. 33). Care is one of the moral qualities that is central to human life. The four discrete yet interconnected phases of care: caring about, taking care, caregiving, and care receiving, which construe the linear process of care: "moving from awareness and intention to actual practice and response" (Kleinman \& Geest, 2009, p. 160). These phases correspond to the four ethical qualities of care: attentiveness, responsibility, competence, and responsiveness (Tronto, 2013, pp. 34-36). Affirming the need to acknowledge

This Open Access article is published under a Creative Commons Attribution Non-Commercial 4.0 International License (http://creativecommons.org/licenses/by-nc/4.0/), which permits non-commercial re-use, distribution, and reproduction in any medium, provided the original work is properly cited. For citation use the DOI. For commercial re-use, please contact editor@rupkatha.com. 
care as a political reality, Tronto observes: "For a society to be judged as a morally, admirable society, it must, among other things adequately provide care for its members and its territory" (1993, p. 126).

In contrast to Tronto's conception of care as a social good, the American philosopher Milton Mayeroff's essay, On Caring (1971), defines care as a personal obligation and self-fulfilling act that stands in contrast to 'power.' In his view, "To care for another person, in the most significant sense, is to help him grow and actualize himself... caring is the antithesis of simply using other person to satisfy one's own need" $(1971$, p. 1). In other words, the moral obligation to care for the other is not forced upon a person, instead the other is "experienced as both an extension of myself and as separate from me" (Kleinman \& Geest, 2009, p. 160). As such, care is a "morally appropriate reaction to other's needs" (Clark, 2005, p. 142). The ethical act of responding to other's needs is at the heart of care. Perceived as a primary human quality, caring includes activities and emotions that are necessary to sustain life. However, the quality of care depends on how the care receiver responds to it. Evidently, Patricia Benner et al observes: "Caring is not dependent on what I do to you, but on how you receive or respond to it" (as cited in DeFalco, 2020, p. 34).

\section{Miscellaneous Conceptions of Caregiving}

Caregiving assumes different meanings across cultures, disciplines, and environments. As Kleinman in his article Catastrophe and Caregiving: The Failure of Medicine as an Art observes: "caregiving is configured by economist as "burden," by psychologists as "coping," by health services researchers in terms of social resources and health care costs and by physicians as a skill" (2008, p. 23). Caregiving is also an indelible part of every religion and culture. For family, professionals, and sufferers themselves, caregiving aid in "the amelioration of pain and suffering" (Kleinman, 2012. p. 1550). In both formal and informal sites, acknowledgement of sufferer's personhood, their condition, and struggle are recognized as the most basic act of caregiving. Nonetheless, caregiving has emotional and technical constituents (Kleinman, 2009, p. 161). While the former makes up a caregiving relationship that entails emotional qualities such as attachment, concern and dedication, the latter refers to the practical assistance provided for the ill or disabled care receivers. In other words, the emotional and technical/ practical aspects of care complement each other. The experience and understanding of caregiving activities vary among different settings. Therefore, the activity of care in a particular setting can be understood listening and observing the actions of people who are directly involved in caring activities (Kleinman, 2009, p. 160). Settings of care can be broadly categorized into three: formal, informal (institutions of care) and medical establishments. Utilising the affordances of comics, graphic medicine visualises and articulates the nuances of the caregiving process in these settings. However, before such an analysis it would be instructive to outline a short history of graphic medicine and a brief overview of the selected graphic memoirs.

\section{Graphic Medicine: Definitions and Scope}

Graphic medicine, an emerging interdisciplinary field of academic study, is "the intersection of the medium of comics and the discourse of healthcare." (Czerwiec et al., 2015, p. 1). The medium's potential to articulate complex issues promotes it as "an ideal way of exploring taboo and forbidden areas of illness and health care" (Czerwiec et al., 2015, p. 3). In his 2016 review of Graphic 
Medicine Manifesto, Venkatesan refers Graphic medicine "as an emerging area of interdisciplinary field which explores comics' distinctive engagement with and performance of illness experience" (p. 93). The interdisciplinary field functions as a novel mode to address illness and those "aspects of social experience that escape both the normal realms of medicine and the comforts of canonical literature" (Squier, 2008, p. 130).

The verbo-visual medium of comics gives "voice to the unsettling worries and concerns that may be difficult to articulate through words alone" (Green, 2015, p. 774). Predominantly autobiographical, the graphic medical narratives illuminate experiential details of physicians, caregivers, and patient and thus "provide[s] an idiosyncratic embodiment of voices that are culturally silenced" (Venkatesan and Peter, 2019, p. 191). Seminal graphic memoirs such as Sarah Leavitt's Tangles (2010), Ian Williams' The Bad Doctor (2014), David B's Epileptic (1996) and Georgia Webber's Dumb: Living Without a Voice (2018), Ken Dahl's Monsters (2009) explore diverse issues such as challenges of caregiving, doctor-patient relationship, medical negligence, among others.

\section{Texts and Contexts}

Sarah Leavitt's Tangles: A Story about Alzheimer's, My Mother, and Me is a graphic memoir about the author's caregiving experiences to her mother, Miriam (referred to as Midge in the text). Most of the caregiving activities in the graphic narrative take place in the domestic setting. Leavitt chronicles her caregiving experiences and archives her mother's life before and after the illness in precise episodes. As DeFalco (2015) claims, Leavitt's use of non-sequential episodic style, a characteristic of "loiterature," encourages the readers "to linger over particular frames or pages" (p. 236). Such stylistic choice facilitates the delineation of how informal caregiving becomes more pronounced during the periods of illness. According to Leavitt's candid summation, she "created this book to remember her (Midge) as she was during her illness, the ways in which she was transformed and the ways in which parts of her endured" (n. p). Depicting her caregiving experiences in raw honesty, Leavitt unfolds the largely unrecognized aspects of informal caregiving.

Intended as a "personal memoir" (Mack, 2004, p. xi), Stan Mack's a dark-humorous cancer tale, Janet \& Me: An Illustrated Story of Love and Loss, is apportioned into ten chapters excluding a prologue and an epilogue. Combining prose and pictures, Mack affords the story an approach distinct from typical comics format. The illustrations often demonstrate the contents of the prose. Written in an episodic style, the graphic narrative "explores the everyday, unexpected pain full details that plagued us (Janet and Stan) as the disease took hold and then worsens" (p. 11). Apart from demonstrating the sufferings that result from cancer, the graphic narrative yields insights into "human-made cancer," such as insurance and billing issues that cause Janet "acute and unnecessary stress" (p. 33). While visualizing their subjective and medical experiences in the "cancerland" (p. 60), Mack also recalls how the hostile hospital environment, that is "busy big mess and even dangerous" (p. 87), fails them.

Joyce Farmer's Special Exits: A Graphic Memoir chronicles the final years of Lars Drover and Rachel Drover (father and stepmother of Laura). Apportioned into fourteen chapters, the graphic memoir depicts the everyday caregiving and care receiving experiences of Laura (Farmer's 
alter ego), Rachel, and Lars in painstaking detail. According to Farmer, the book is "about two people dying" (Campbell, 2011, n. p). Besides depicting her caregiving experiences, Farmer unfolds the mistreatments that her stepmother suffers due to failed care in the nursing home. As such, Farmer brings to relief the camouflaged aspects of institutional care. The three graphic caregiving memoirs: Tangles, Janet \& Me, and Special Exits, communicate the distinctive nature of caregiving in formal, informal, and biomedical settings, respectively.

\section{"Take a deep breath and just do what needs to be done": Informal Caregiving}

In informal settings, caregiving is recognized as an essential part of relations that facilitates families and friendship networks cope up with precarious conditions among their own (Kleinman, 2012, p. 1550). Put boldly, informal caregiving is an unpaid and 'high-quality care' predominantly provided by untrained family members to a person in precarious state within the family circle. The normative cultural perception about informal care as an "offering" (Dragojlovic \& Broom, 2018, p. 44) from one person to the other, overlooks the labour involved in the same. Put boldly, in informal settings the "labour of caring" is viewed as "labour of love" (Dragojlovic \& Broom, 2018, p. 44). Sarah Leavitt's graphic caregiving memoir Tangles centers primarily on the author's caregiving experiences to her Alzheimer's-afflicted mother, Midge. Unfolding both the comforting and disquieting aspects of informal care, the graphic narrative lays bare the everyday labor demanded by informal caregiving.

Unlike professional caregivers, the informal caregivers move "from the status bystander to engaged sharer, often helping the sufferer to process it all. It is this mutual engagement that caregiving becomes an enriching human process" (Kleinman, 2019, p.156). In other words, over time, the informal caregiver becomes an extension of the care receiver's self. As the illness robs Midge's capacity to carry out her basic care necessities, Sarah becomes the extension of her mother's self. Underscoring the lack of options available for informal caregivers during the gruelling moments of caregiving, Sarah states: "There are moments when you have a choice: fall apart or take a deep breath and just do what needs to be done" (p. 60). Sarah's unremitting caregiving becomes particularly pronounced when Midge is overshadowed by her illness. When she discovers that Midge "couldn't recognize shirt, dirt and shame" (p. 60), she pitches in to help Midge despite her detesting plight. The panel pictures Midge sitting inside the bathtub, holding a washcloth close to her nose with a radiant expression on her face.

The captions inside the panels accentuate the enormity of Midge's plight from the caregiver's perspective thus: "She was dipping her washcloth in the water and rubbing it over her skin. She had no sense of smell, true. But she could see. She just couldn't recognize. Couldn't recognize shit, dirt and shame" (p. 60). Subsequently, the succession of nine short panels with captions: "Drain the tub. Spray the water hard so everything goes down. Rinse, Wash. Rinse. Take her hand. Let her dry herself. Help her with her nightie. Tuck her into a warm dry bed. Turn out the light" (p. 60), indicate the orders that Sarah gives herself to help her mother. The images in each of these panels visually demonstrate these captions. Strikingly, despite Sarah's (invisible) presence, the images in these panels picture only Midge and the duties done for her. This way of representation indicates the informal caregiver's stance to put her preferences aside to prioritize the care receiver's need. In the last panel, Sarah experiences a new feel of "loneliness" and 
5 | Spaces of Care and Graphic Medicine

"strength" (p. 60). As such, the graphic narrative unfolds both the benefits and burdens of informal caregiving.

Apart from taking care of Midge and scrambling to find new ways to cope, the family caregivers painstakingly witness the corrosive effects of an illness that tears away Midge from the rest of her family and herself. According to Leavitt, "Alzheimer's disease tore her away from us and from herself in a cruel, relentless progression of losses" (n.p). Consequently, Midge, a formerly primary caregiver who played a role of a caring mother, and supportive wife and sister, becomes "a list of needs: BATH, CLOTHES, BRUSHTEETH, WALK, FOOD, ETC." (p. 85) as she cascades into a progressive cognitive deterioration. Depicting both the "competence and helplessness" (DeFalco, 2015, p. 234) of informal caregivers, this graphic narrative demonstrates the ambivalent nature of informal care. Elsewhere, Sarah says that, "Sometimes I felt like the calmest, most capable nurse. Other times I thought I might throw up from the smell of urine and shit" (p. 110). Through representing informal caregiving as at once "rewarding and frustrating, emotional and physical" (DeFalco, 2015, p. 237), this graphic narrative dismantles the normative socio-cultural assumptions about informal caregiving.

\section{"In a hospital setting one becomes a chart rather than a person": Hospital Care}

In healthcare, care is predominantly relegated to "understanding the disease process and high technology treatments" (Kleinman \& Geest, 2009, p. 162). Physicians pay scant attention to the illness experience of the patient and "treat caregiving as a foreign or a distant, nearly forgotten relative" (Kleinman, 2019, p. 148). Put boldly, the caregiving that is central to the practice of medicine "has gone from bad to worse" (Kleinman, 2019, p. 101). Rather than addressing the patient's existential core, the medical professionals engage in care on the "most superficial and mechanical level" (Kleinman, 2019, p. 69). Among the three chosen graphic memoirs, Mack's Janet \& Me, trenchantly illustrates the technical nature of biomedical care. According to Mack's candid summation, the graphic narrative intends to explore "the everyday, unexpected, painful details" that plagues Janet and Stan as the disease continues its implacable course and "the human problems that the doctors would have us believed stopped short of their doors" (p. 11).

When Stan and Janet visit the hospital for biopsy results, the surgeon abruptly conveys the life-changing information, which is "too new, too scary, too shocking to face directly" in a "busy and narrow corridor" (p. 15) and then rushes off. This illustration encapsulates the couple's shock and confusion at the news and the doctor's unnerving abruptness. The sparsely drawn illustration depicts Stan and Janet standing near the cashier's office, facing the readers, with squiggly lines emanating above their heads, which are symbolic of their dismay and bewilderment at the "bad news" and the doctor's apathetic attitude towards the diagnosis. Commenting on their estranging clinical encounter with surgeon, Mack states, "We could hardly absorb the diagnosis. We left the hospital looking for a lighthearted way to talk about the information" (p. 15). The surgeon's callous disclosure of the diagnosis: "It's cancer" (p. 15), followed by the treatment option for surgery creates a deeply unsettling atmosphere for the protagonists. This inadequate medical response to the seemingly serious illness acts as a prelude for the couple's ensuing reprehensible medical experiences. 
As the graphic narrative progresses, caregiving becomes increasingly peripheral to the medical treatment that Janet receives. The graphic memoir lays bare how biomedicine disregards the experiential aspects of Janet's illness. By categorising Janet's test results, which confirm the presence of a virulent form of cancer, into "the good news" (p. 59) and "the bad news" (p. 59), Laura, Janet's oncologist, marginalizes the former's human condition. Laura says "First, the good news, Janet. The cancer is not in any organs. The tests show it's only in the bones... Now the bad news. The treatment you've been on for six months isn't working" (p. 59). She also adds that the cancer has spread to Janet's left arm, left leg, skull, spine, and pelvis (p. 59) and immediately shifts the emphasis to schedule chemo to "treat all the area at once" (p. 59). This scene evinces the apathy of the oncologist to Janet's medical condition and promulgates a subtle criticism on the absence of empathy towards the patient's emotional landscape. Elsewhere, conceding the seriousness of Janet's illness, Danny, a new intern says: "She had a diffuse metastasis to the skeleton and the general sense is that it's rare for the patient to get better" (p. 76). In contrast to Laura's insouciance to Janet's diffused metastasis, Stan expresses the cold touch of Janet's diagnosis on him thus: "The word skull really shook me" (p. 60). The concurrent mentions of the two disparate perspectives from the informal caregiver and the biomedical practitioner about the test results metaphorically lay bare the absolute difference of how the former and latter engage in the caregiving process. While Laura, prioritizing objective clinical medicine, mechanically tries to impose care through medical treatment, Stan's reactions indicate his engagement with Janet's condition at a personal and emotional level. Elsewhere, concerning Laura's superficial and ambiguous response, Stan writes that "her own words seemed studied and non-specific, always leaving us with the handholds on hope" (p. 19). Eventually, when Laura confirms that Janet's cancer is incurable, the former still insists on medications to repair Janet's worsening health. Here, care itself "might be (becomes) an instance of not caring" (Noddings, 2013, pp. 3-4). Laura's "ambiguous" (p. 80) response, her reluctance to admit Janet's declining health by continually thrusting Janet into different medical regimens, her nonchalance to "talk honestly" (p. 81) about "the proverbial invisible elephant" (p. 81) (i.e., Janet's fate) that hurts the couple and her failure to answer Stan's and Janet's messages on her declining health thrust the couple into the throes of sufferings. Elsewhere, alluding to the doctor's unsupportive stance and hesitancy to talk about the patient's real condition, Paul, "the then- executive director of Jacob Perlow Hospice", which provided terminal care to Janet, says "Some keep doing chemo as the body is being taken to the undertakers" (p. 146).

The graphic narrative also brings Janet's plight into relief, especially when she is subjected to unsympathetic treatment by the medical establishment. The instances of care in the hospital setting become the source of "pathogenic vulnerability", such as when the response intended to ameliorate vulnerability has the paradoxical effect of exacerbating existing vulnerabilities and itself becomes the cause of suffering (Mackenzie et al., 2013, p. 9). Mack lays bare the predicament that Janet undergoes when she is admitted in the hospital as a result of her "extreme hip pain" (p. 87). Referring to the obnoxious hospital setting, Stan says: "Hospital, supposedly centers of safety and healing, could be hazardous to your health" (p. 87). Descriptions such as of a woman lying in her own waste and moaning for help in the bed next to Janet indicate the transition of the setting of care into a storage space for patients. The negligence with which the medical professionals swipe the blood when "the needle broke and my (her) blood poured all over the bed" and the "mean, 
sloppy and uncaring" (p. 87) nurses and attendants suggest the dehumanizing atmosphere that a hospital setting provides to the patient. These instances suggest that there is a stark demarcation in biomedicine between the objectivity of science and subjectivity of the illness. In these examples, the subjective aspects of the patients are stripped away, and care is restricted to medical interventions. The other biomedical oversights in Janet \& Me include the hospital setting that treats a patient as a chart rather than a person (p. 75), and the detached professional arrogance of medical professionals who insist on following "doctor's orders" (p. 76) and keep taking blood from Janet's poor veins till she was crying in pain" (p. 75).

\section{"Everything Hurts": Institutional Care}

"Institutional care is provided within a congregate environment designed to meet the functional, medical, personal, social, and housing needs of individuals who have physical, mental/ developmental disabilities" (Galik, 2013, p. 1079). In Special Exits, Laura navigates the bureaucratic institutional placement, which eventually harms and weakens Rachel further. The images of the nursing home's employees make their negligence, disinterest, and detachment from their duties obvious. The panels depicting the staff informing Laura over the phone about Rachel's fall from the bed reveal their disengagement and nonchalance towards Laura's queries about her stepmother's accident. The bored and distracted employee wears an empty smile, holds a sandwich in her hand, and plays a game of crosswords while conversing with Laura about the potentially serious incident. When the latter anxiously enquires about the specificities of Rachel's condition and the cause of the accident the member of staff, tapping a pen on the table with a bored face, casually responds to that she does not know the cause of Rachel's fall. When Laura requests the staff in the nursing home to tell her the cause of her stepmother's fall, the latter declares that she is unaware of the cause: "I don't. I found her on the floor beside her bed." ( $p$. 138). Later, when she questions the employee if the bed rails were up, she unapologetically says, "No, ma'am. They (bed rails) was down. I just left her for a minute" (p. 138). The responses of the nursing home employees not only demonstrate their detachment and apathy but also indicates the marginalization of caregiving in the institutional setting.

As opposed to the uplifting and safer atmosphere of the domestic spaces, the nursing home turns out to be a precarious setting that sabotages Rachel's health. Referring to her state of health prior to her admission to the nursing home, Dr. Wilshire says: "She is healthy! She can live for years! Your father obviously takes good care of her" (p. 135). Similar to Leavitt's Tangles, the scenes of informal caregiving reveal both the pleasure and burden of informal care. At home, Laura, and Lars, despite his old age, take care of Rachel. She becomes increasingly dependent on the caregivers as a result of her disabilities and her blindness caused by old age and glaucoma. However, her needs and endless requests for assistance are carefully addressed by the caregivers without fail. For instance, despite his hesitation, Rachel's repeated demands for food and drink: "Lars, do we have anything to eat or drink now?" (p. 116) are promptly attended to by Lars. Elsewhere, implying Rachel's demands and his caregiving burden, Lars says: "She's been more alert since we started her thyroid pills again. But now she's more demanding. It was easier on me when she slept all the time. I'm not saying should stop the pills" (p. 60). Nonetheless, Lars and Laura decide to admit Rachel to the nursing home as her needs exceed their capacity to handle them. Laura says: "Dad can't handle any more and he has put me in charge... she needs to be 
cared for outside their home." (pp. 135-136). However, soon after Rachel's fall from the bed, Laura resents her decision to admit the former in the institutional placement, she says, "My God, what have I done to her? She fell many times at home. It was fourteen inches to the soft carpet" ( $p$. 139). The callousness of the nursing home staff and their failure to carefully monitor Rachel subjects her to mortal risk and harm. Eventually, Rachel is belatedly (a week later) diagnosed with a "broken hip" (p. 139), which is later pinned with surgery that causes immobilisation and permanent pain. Thereafter, she becomes largely inert, surrounded by medical devices, visually diminished, static and in pain. Elsewhere, referring to the nursing home facility that fails to take good care of Rachel, Laura says to the director of the nursing home: "My mother was blind but in good health when she came here... It is very clear that your facility is responsible for her situation... My otherwise healthy mother has had nothing but pain and is now near death" (p. 147). The other institutional oversights include staff who neglect to feed Rachel, despite a notice behind her saying "Blind-Must be Fed" (p. 147), and employees who conduct unnecessary diabetes tests, on doctor's orders, that injure her fingers. Instead of being a place for safety and help, the sterile institutional site reduces Rachel into a kind of animate object without feelings or actions of care (DeFalco, 2015, p. 234).

\section{Conclusion}

In formal, informal, and hospital settings caregiving plays a crucial role in alleviating people's pain and suffering. However, the labour of caring in each of these settings is disproportionately divided. Through depicting both the challenging and the comforting aspects of informal care, the graphic narrative resists the idealization of the same as the "labour of love." Despite the benevolent intention to care for the sufferer, the caregiving burden overwhelms the family members themselves. The verbo-visual medium captures the particularities of informal caregiving in viscerally engaging ways.

Differently, in the hospital setting the emotional and practical aspects of caregiving are increasingly marginalized. Caregiving in this setting is constrained to medical prescriptions, technology, and diagnosis. Instead of addressing the patients' subjective concerns, the biomedical procedures ratchet up the sufferer's frustration and anxiety. Exposing the horrors that the patients face in the institutional setting of care, the graphic narrative brings into relief how the carereceivers demands are considered as an afterthought. Despite being the centers of safety that should aid the care receiver to carry out his/her necessities, the setting pay scant attention to the person's physical and emotional sufferings. In essence, the graphic medical narratives do not create an opposition among informal, formal, and biomedical settings of care but affirms the undeniable attention that the sufferers receive in an informal setting. As such, the true meaning of caregiving is ingrained in informal sites of care.

\section{References}

Benner, Partricia, et al. (1996). Introduction. In P. Benner, S. Gordon, \& N. Noddings (Eds.), Caregiving: Readings in knowledge, practice, ethics, and politics, (pp. vii- xvi). Philadelphia PA: University of Philadelphia Press. 
9 | Spaces of Care and Graphic Medicine

Caduff, C. (2019). Hot chocolate. Critical inquiry, 45(3), 787-803. doi:10.1086/702591.

Campbell, J. (2011, February 08). Farmer discusses "Special Exits". Retrieved April 30, 2021, from https://www.cbr.com/farmer-discusses-special-exits/.

Czerwiec, M. K, et al. (2015). Graphic medicine manifesto. Pennsylvania: The Pennsylvania State UP

DeFalco, A. (2020). Towards a theory of posthuman care: Real humans and caring robots. Body \& Society, 26(3), 31-60. doi:10.1177/1357034x20917450

Dragojlovic, A., \& Broom, A. (2018). Bodies and suffering: Emotions and relations of care. Abingdon, Oxon: Routledge.

Farmer, J. (2014). Special Exits: A Graphic Memoir. Seattle: Fantagraphics Books.

Fisher, B., and Tronto J. C. (1990). Toward a feminist theory of caring. E. K. Abel \& M. K. Nelson (Eds.), Circle of care: Work and identity in women's life (pp. 35-62). Albany: State university of New York.

Galik E. (2013). Institutional Ccare. M.D. Gellman \& J. R. Turner (Eds.) Encyclopedia of behavioral medicine (pp. 1079-1080). New York: Springer. https://doi.org/10.1007/978-1-4419-1005-9_1424

Green, M. J (2015). Comics and Mmedicine: Peering into the process of professional identity formation. Academic Medicine. 90 (6), 774-9.

Held, V. (2006). The ethics of care: Personal, political, and global. Oxford: Oxford University Press.

Kleinman, A. (2006). What really matters: Living a moral life amidst uncertainty and danger. New York: Oxford University Press.

Kleinman, A. (2007). The bioculture of caregiving: A commentary on "biocultures". New Literary History, 38(3), 593-599. doi:10.1353/nlh.2007.0043

Kleinman, A. (2008). Catastrophe and caregiving: The failure of medicine as an art. The Lancet, 371(9606), 22-23. doi:10.1016/s0140-6736(08)60057-4

Kleinman, A. (2012). Caregiving as moral experience. The Lancet, 380(9853), 1550-1551. doi:10.1016/s01406736(12)61870-4

Kleinman, A. (2020). The soul of care: The moral education of a husband and a doctor. New York: Penguin Books.

Kleinman, A., \& Geest, S. V. (2009). Care in health care: Remaking the moral world of medicine. Medische Antropologie, 21(1), 159-168.

Leavitt, S. (2012). Tangles: A story about Alzheimer's, my mother, and me. New York: Skyhorse Publishing.

Mack, S. (2004). Janet \& me: An illustrated story of love and loss. New York: Simon \& Schuster.

Mackenzie, C. (2014). Vulnerability: New essays in ethics and feminist philosophy. New York: Oxford Univ. Press.

Mayeroff, M. (1971). On Ccaring. New York: Harper Collins.

Miller, S. C. (2005). Need, care and obligation. In Soran Reader (Eds.), The philosophy of need, (pp. 137-160). Cambridge: Cambridge University Press.

Noddings, N. (2013). Caring: A relational approach to ethics and moral education. Berkeley: University of California Press.

Squier, S. M. (2008). Literature and medicine, Future tense: Making it graphic. Lit Med, 27 (2), 124-52. 
Tronto, J. C. (1993). Moral boundaries: A political argument for an ethic of care. London: Routledge.

Tronto, J. C. (2013). Caring democracy: Markets, equality, and justice. New York, London: New York University Press.

Venkatesan, S. 2016. Graphic Medicine Manifesto, by M. K. Czerwiec, Ian Williams, Susan Merrill Squier, Michael J. Green, Kimberly R. Myers, and Scott T. Smith. Journal of Graphic Novels Comics, 7 (1), $93-$ 94

Sathyaraj Venkatesan is Associate Professor of English in the Department of Humanities and Social Sciences at the National Institute of Technology, Trichy (India). He is the author of six books and over ninety research publications that span African American literature, health humanities, graphic medicine, film studies, and other literary and cultural studies disciplines. He is most recently co-author of Gender, Eating Disorders and Graphic Medicine (Routledge, 2020) and India Retold (2021).

Livine Ancy A is a PhD Research Scholar in the Department of Humanities and Social Sciences at the National Institute of Technology, Trichy (India). Her research concentrates on graphic medicine, visual care studies and literary health humanities. 